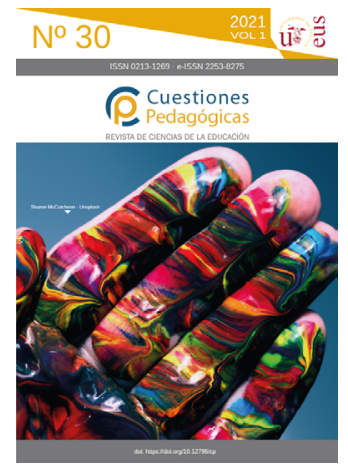

Diagnosis intercultural con perspectiva

de género en escuelas.

Estudio de caso múltiple

Intercultural analysis with a gender perspective in schools

Multiple case study

Recibido: 28/02/2021 | Revisado: 29/04/2021 | Aceptado: 08/06/2021 |

Online first: 10/06/2021 | Publicado: 01/07/2021

\title{
Rocío Rodríguez-Casado
}

Universidad Pablo de Olavide (España)

mrrodcas@upo.es

https://orcid.org/0000-0002-8263-6154

\section{Teresa Terrón Caro}

Universidad Pablo de Olavide (España)

mttercar@upo.es

https://orcid.org/0000-0003-4177-0346

\begin{abstract}
Resumen: Desde una visión socioeducativa, los estudios de casos múltiple permiten explorar al detalle colectividades pluriculturales que necesitan respuestas institucionales y profesionales para garantizar una adecuada gestión de los desafíos pedagógicos que continúan inmersos en las instituciones educativas, como son la diversidad cultural y de género. En este sentido, nos cuestionamos si hoy, en las escuelas del siglo XXI, se logra transversalizar un enfoque real y efectivo basado en la interculturalidad con perspectiva de género para dejar atrás la escuela tradicional, que encubre tanto conductas como actitudes racistas y sexistas. En la presente investigación, a través de un sistema metodológico mixto y desde una mirada comparativa, nuestra finalidad es analizar las acciones interculturales con perspectiva de género desarrolladas en cuatro centros educativos públicos, dos de Sevilla (España) y dos de Salta (Argentina), para conocer qué se entiende por dicho enfoque y si desde el profesorado se considera pertinente incorporar y aplicar en sus entornos educativos. Dichas instituciones educativas son seleccionados por criterios muestrales. Los resultados obtenidos, tanto de las encuestas al profesorado como de los grupos de discusión a las comunidades educativas sobre las prácticas desarrolladas para la atención a la diversidad cultural y la perspectiva de género, muestran la necesidad de formación especializada en esta materia, la colaboración de agentes socioeducativos en el sistema educativo y la renovación e innovación de las normativas educativas institucionales a las nuevas demandas sociales y culturales, que influyen directa e indirectamente, para alcanzar el éxito educativo en su máxima expresión.
\end{abstract}

Palabras clave: diversidad cultural, educación intercultural, coeducación, escuela primaria, estudio de caso.
Abstract: From a socio-educational perspective, the multiple case studies allow the exploration of multicultural communities in detail that need institutional and professional responses to guarantee an adequate management of the pedagogical challenges that continue to be immersed in educational institutions, such as cultural and gender diversity. In this sense, we question whether today, in the schools of the 21st century, it is possible to mainstream a real and effective approach based on interculturality with a gender perspective to leave behind the traditional school, which conceals both racist and sexist behaviors and attitudes. In this research, through a mixed methodological system and from a comparative perspective, our purpose is to analyze intercultural actions with a gender perspective developed in four public educational centers, two in Seville (Spain) and two in Salta (Argentina), to find out what is meant by said approach and if teachers consider it pertinent to incorporate and apply it in their educational environments. These educational institutions are selected by sampling criteria. The results obtained, both from the teacher surveys and from the discussion groups to the educational communities on the practices developed for the attention to cultural diversity and the gender perspective, show the need for specialized training in this matter, the collaboration of Socio-educational agents in the educational system and the renewal and innovation of institutional educational regulations to the new social and cultural demands, which directly and indirectly influence, to achieve educational success in its maximum expression.

Keywords: cultural diversity, intercultural education, coeducation, primary school, case study. 


\section{La Interculturalidad con Perspectiva de Género en el Ámbito Educativo Reglado}

¿La interculturalidad con perspectiva de género es una realidad que influye y confluye en todo sistema educativo? Para dar con coherencia una respuesta es necesario que reflexionemos sobre lo que se dice y lo que se pone en práctica, dado que, ante la realidad pluricultural indiscutible en las instituciones educativas, esta investigación se dirige hacia la efectividad real de la interculturalidad con perspectiva de género como modelo socioeducativo de gestión para la diversidad cultural y la perspectiva de género en las aulas de los centros escolares en los que hay que intervenir de manera inclusiva, integradora, igualitaria y equitativa para con todo el alumnado e involucrando a toda la comunidad educativa (profesorado, equipo directivo, otros/as profesionales socioeducativos y familias ${ }^{1}$ ). Pues, la educación intercultural y la perspectiva de género no es un hecho o necesidad de ahora, sino un planteamiento teórico-práctico con carácter pedagógico que ha ido evolucionando y visibilizándose, aún más, en el ámbito educativo, y desde el que debemos abandonar ideologías basadas en la diferenciación cultural y de género, tal y como recogen en sus trabajos multitud de autorías (Besalú, 2002; Aguado, 2004; Álvarez, 2008; Ariño, 2009; Rodríguez Navarro et al. 2011; Tomé, 2011; Osuna, 2012; Subirats, 2016; Ruiz, 2017).

La educación no tiene como única finalidad crear solamente buenos/as estudiantes, sino estudiantes capaces de reproducir su propio pensamiento crítico y que sean conscientes de las capacidades, habilidades, destrezas y competencias que poseen para empatizar hacia la justicia social y luchar en contra de situaciones conflictivas, desiguales y discriminatorias, como son las generadas por cuestiones culturales y de género.

En este sentido, nos preguntamos ¿qué caracteriza una práctica pedagógica intercultural con perspectiva de género para alcanzar un espacio educativo justo y equitativo? Esto requiere, por un lado, de una reflexión acerca de la cultura organizativa (filosofía escolar) de los centros escolares, sobre sus valores y actitudes, así como sobre la manera en la que se construye el conocimiento de la realidad sociocultural desde la perspectiva de género. Y, por otro lado, conocer la práctica real basada en la interculturalidad con perspectiva de género para que se genere una armonía real y holística en las escuelas (Rodríguez, 2017).

La gestión de la diversidad cultural y la perspectiva de género en los centros educativos constituye dos de los principales desafíos a lo que se enfrenta todo sistema educativo reglado, independientemente del contexto en el que nos encontramos, persiguiendo modelos que permiten el adecuado desarrollo socioeducativo del alumnado y una convivencia enriquecedora de toda la comunidad educativa. Por ello, la interculturalidad con perspectiva de género conforma una misión enfocada a la apertura hacia la transformación socioeducativa de las escuelas a partir del desarrollo del aprendizaje cooperativo y constructivo del conocimiento en igualdad real y efectiva entre los y las menores, ya que seguimos evidenciando cuestiones pedagógicas vinculadas a ciertas concepciones multiculturalistas y sexistas que llevan hacia el extremo de las diferencias, culturalizando las desigualdades sociales, culturales y de género, entre otras, y con ello, transgrediendo el fin de la educación basado en valores democráticos, cívicos y de derecho (Rodríguez, 2018).

Defender la transformación socioeducativa y cultural para las escuelas de hoy no es un proceso fácil, pero sí indispensable para seguir avanzando en cuestiones educativas, sociales y culturales de las que emergen y se insertan toda comunidad educativa que trabaja hacia el respeto efectivo, la inclusión educativa y la convivencia escolar por y para alcanzar la igualdad de todos/as sus componentes. Trabajar desde este enfoque socioeducativo significa "fortalecer la democracia y la ciudadanía participativa a través de una educación integral guiada por valores como la solidaridad, la igualdad y la justicia" (Ayuste, 2011, p. 19).

1 En esta investigación entendemos por comunidad educativa a todas las personas inmersas en una institución educativa, desde el alumnado, profesorado, personal de mantenimiento y limpieza, otros/as agentes socioeducativos (pedagogos/as, orientadores/as, mediadores/as, monitores/as...) hasta las familias. Sin embargo, para el desarrollo de los grupos de discusión no se pudo contar con los menores por su protección tanto administrativa-judicial, como personal. 
De acuerdo con las aportaciones de diferentes autorías (Borrero, 2012; Ballesteros et al. 2014; Subirats, 2016; Rodríguez, 2017) se ha diversificado los términos de interculturalidad y perspectiva de género en las escuelas a raíz de investigaciones y otras experiencias docentes didácticas realizadas para atender a la diversidad del alumnado (ya sea cultural, social, de género, funcional...), debido a los continuos cambios y avances sufridos en nuestra sociedad y a nivel mundial, llámese globalización (procesos migratorios, avances tecnológicos, estilos de vida, quebrantamiento de la economía...). Sin embargo, aún existen cuestiones pedagógicas, desde la visión del profesorado, ligadas a estereotipos y prejuicios culturales y de género en las instituciones educativas que se siguen transmitiendo, incluso, de forma inconsciente, insidiosa, casi en la invisibilidad y naturalidad, así como con mucha sutilidad (Barbieri y Ferede, 2020).

En este sentido, uno de los primeros pasos para deconstruir situaciones de discriminación y desigualdad en las escuelas es reconociendo que existen, y es ahí cuando podremos avanzar hacia la interculturalidad con perspectiva de género, es decir, "ayudar a cuestionar las situaciones estructurales y las condiciones que permiten que algunas culturas dominen sobre las otras, que unos grupos humanos sean preferidos sobre otros, que son, a su vez, rotulados como diferentes e inferiores" (Medina, 2019, p. 205).

A tenor de lo expuesto, mostramos los resultados más significativos ${ }^{2}$ sobre la concepción y opinión que posee el profesorado y la comunidad educativa, compuesta por miembros del equipo directivo, miembros del equipo docente, otros/as profesionales socioeducativos y familias, de los centros escolares seleccionados, con respecto a la necesidad e importancia de implantar acciones interculturales con perspectiva de género en instituciones educativas.

De esta forma, podemos conocer, entender y valorar qué se entiende por dicha temática y qué importancia alcanza, por parte de los y las agentes socializadores implicados/as (profesorado y comunidad educativa) tanto dentro como fuera de las escuelas, el hecho de trabajar desde un enfoque intercultural con perspectiva de género.

\section{Abordaje Metodológico}

El presente estudio de caso múltiple comparativo sustenta un diseño de investigación explicativo, descriptivo, interpretativo y evaluativo, cuya finalidad es conocer, entender y valorar la concepción y opinión que posee el profesorado y la comunidad educativa de los centros escolares seleccionados con respecto a la necesidad e importancia de poner en práctica acciones interculturales con perspectiva de género dentro del ámbito educativo.

Así, a la hora de investigar en el campo de las Ciencias Sociales, cuya realidad es cambiante y compleja, es de gran utilidad implementar una metodología mixta de investigación como vía fundamental para que el equipo de investigadoras pueda acercarse a su objetivo de estudio y obtener el conocimiento veraz de la realidad según sus necesidades y expectativas, concretamente, de las instituciones educativas seleccionadas (Del Canto y Silva, 2013).

En esta ocasión, y para alcanzar la finalidad de este trabajo, a través de una metodología mixta, implementamos una encuesta, a nivel cuantitativo, cuyo cuestionario, con un índice de fiabilidad de 0,971 de Alfa de Cronbach, dirigimos al equipo docente de cada centro escolar seleccionado.

$Y$, a nivel cualitativo, diseñamos un guion de preguntas en consonancia al diseño del grupo de discusión, de no más de 12 personas con diferentes perfiles (profesorado, familias y/u otros agentes educativos que trabajan dentro de la escuela), con la finalidad de conocer la percepción y opinión sobre

2 Tesis Doctoral titulada "Diversidad cultural y perspectiva de género en centros educativos de infantil y primaria. Estudio de caso múltiple en Sevilla y Salta" (Rodríguez, 2017). Esta investigación fue premiada con una Beca JPI del Banco Santander en el curso académico 2014/2015 para la realización del trabajo de campo en Argentina. Enmarcada dentro del proyecto de investigación internacional Research staff Exchange scheme (IRSES) "Gendercit: Gender and Citizenship" (2013 - 2017). Coordinado por la Universidad Pablo de Olavide (Sevilla), dentro del subprograma "People" del $7^{\text {a }}$ Programa Marco Europeo de i+dt, financiado por la Unión Europea en el marco del Programa Marie Curie Action (fp7-people-2012-irses), con referencia no 318960. Premio Extraordinario de Doctorado de la Universidad Pablo de Olavide (España). 
las acciones interculturales con perspectiva de género de los y las informantes clave de la comunidad educativa de cada centro escolar seleccionado.

En el desarrollo de esta metodología mixta hemos considerado como estrategias de investigación: la complementación, la combinación y la triangulación (Corbetta, 2007). Asimismo, destacamos un exhaustivo proceso de validación de ambos instrumentos a nivel interno y externo a través del diseño de un Dossier de Validación dirigido a expertos/as relacionados, por una parte, con las temáticas de la investigación, y, por otra parte, con el sistema metodológico, para recibir recomendaciones y mejoras en cuanto al método, la metodología mixta y las técnicas a implementar, teniendo en cuanta la contextualización de la investigación por países de estudio.

La selección de los centros escolares por provincia, Sevilla (España) y Salta (Argentina), se justifica según los criterios del equipo de investigadoras de forma deliberada, no aleatoria, ni probabilística (véase figura 1), así como ajustándose a los criterios muestrales, basados a su vez en parámetros comparativos de acuerdo con los contextos de estudio, que están enfocados en la accesibilidad a los centros educativos, con alumnado mixto y presencia de alumnado de origen extranjero, originario y/o de minorías étnicas, ubicados en las zonas urbanas de las provincias objetivo de estudio y, por último, centros educativos de infantil y primaria con antecedentes sobre prácticas relacionadas con la diversidad cultural y la perspectiva de género ${ }^{3}$.

\section{Figura 1}

Parámetros comparativos del proceso metodológico del Estudio de Caso Múltiple.

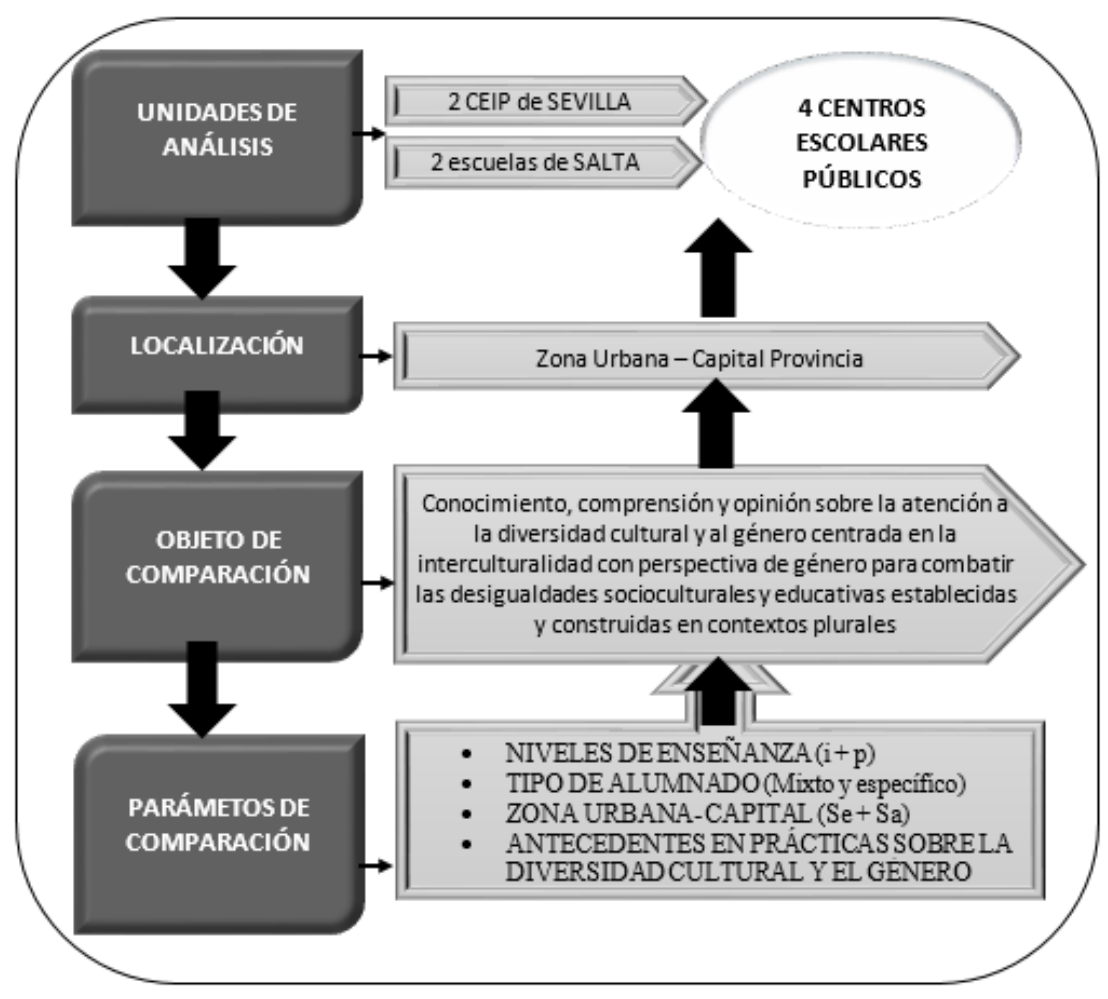

Fuente. Elaboración propia.

Cabe destacar que la mirada comparativa de esta investigación nos posibilita un elemento contextual que enriquece el análisis de los datos obtenidos hacia una valoración más completa y, a su vez, compleja. Por ello, esta comparativa tiene presente tres factores: 1) las unidades de análisis, 2) la temática planteada, determinando una seria de categorías comparativas y 3) los universos, es decir, las zonas contextuales y su historia, Sevilla (España) y Salta (Argentina).

3 La contextualización de esta investigación se enmarca en el proyecto de investigación internacional Research staff Exchange scheme (IRSES) "Gendercit: Gender and Citizenship" (2013 - 2017). Coordinado por la Universidad Pablo de Olavide (Sevilla), dentro del subprograma "People" del 7 a Programa Marco Europeo de i+dt, financiado por la Unión Europea en el marco del Programa Marie Curie Action (fp7-people-2012-irses), con referencia nº 318960. 
Para acabar, recordamos que la finalidad de este estudio de caso múltiple es indagar en las particularidades propias de cada caso escolar seleccionado, relacionadas con la temática de interés, en este caso, sobre las acciones interculturales con perspectiva de género, y por ello, la representatividad no está presente.

[...] la selección de informantes, episodios e interacciones van siendo conducidas por un planteamiento conceptual, no por una preocupación por la representatividad. Para llegar al constructo, necesitamos ver sus diferentes aspectos, en diferentes momentos, en diferentes lugares, con diferente gente. La mayor preocupación es por las condiciones bajo las cuales el constructo o la teoría operan, no por la generalización de los resultados a otros contextos (Miles y Huberman, 1994, citado en Valles, 1999, p. 94).

En definitiva, el estudio de caso múltiple nos posibilita la adquisición de conocimientos fiables, sistemáticos y rigurosos dada la metodología mixta implementada en cada contexto de estudio con el fin de indagar en las complejidades existentes en toda realidad socioeducativa, en nuestro caso el conocimiento, la comprensión y valoración sobre la gestión de la interculturalidad con perspectiva de género en las instituciones educativas seleccionadas para reducir y combatir las desigualdades y discriminaciones por razonas culturales y de género.

\section{Discusión de los Resultados}

En este apartado presentamos desde una mirada comparativa cómo se perciben las acciones interculturales con perspectiva de género por parte de las comunidades educativas y desde la visión del profesorado de cada centro educativo. Cabe recordar en esta discusión empírica la contextualización de las instituciones analizadas. Las instituciones educativas son: las escuelas salteñas (Ca1) Escuela Indalecio Gómez y (Ca2) Escuela Roberto Romero, y en Sevilla los CEIP (Ca3) San José Obrero y (Ca4) Andalucía.

\section{Comparación de la Visión de las Comunidades Educativas ante las Acciones Interculturales con Pers- pectiva de Género}

Los indicadores cualitativos determinados para conocer, entender y valorar qué visión posee la comunidad educativa de cada centro escolar seleccionado sobre las acciones interculturales con perspectiva de género son:

, Consideración del concepto: qué se entiende por interculturalidad con perspectiva de género.

, Acciones desarrolladas en el centro escolar relacionadas con la interculturalidad con perspectiva de género.

> Uso de materiales didácticos para trabajar la interculturalidad con perspectiva de género.

Desde la comunidad educativa de la Escuela Indalecio Gómez (Ca1) de Salta, se entiende la interculturalidad y la perspectiva de género desde la igualdad entre todo el alumnado desde su diversidad cultural y de género. Es decir, se declara que hay que trabajar la desconstrucción de estereotipos y roles determinados por la cultura que solo hacen mantener las desigualdades por ser diferente. Por su parte, la comunidad educativa de la Escuela Roberto Romero (Ca2) de Salta, considera la interculturalidad y la perspectiva de género como una herramienta didáctica para adquirir conocimiento y crear un mayor vínculo con aquel alumnado que se encuentra en una situación de desventaja, problemática y discriminación.

En la provincia de Sevilla, la comunidad educativa del CEIP San José Obrero (Ca3) manifiesta que el trabajo desde la interculturalidad y la perspectiva de género se hace a diario, no es puntual. En este centro escolar se atiende a la diversidad cultural y a la perspectiva de género desde la igualdad, la inclusión, la equidad, la convivencia, el respeto de todo el alumnado, profesorado y familias. En cuanto al CEIP Andalucía (Ca4) no se especifica una respuesta determinada, sino, más bien, que existe bajo entendimiento sobre qué es la interculturalidad con perspectiva de género.

Con respecto a las acciones interculturales y con perspectiva de género, la comunidad educativa de la Escuela Indalecio Gómez (Ca1) de Salta declara que son puntuales, sobre todo relacionadas con la educación sexual, pero que considera muy importante este enfoque para trabajar desde y con las 
diferencias. En la misma línea, la comunidad educativa de la Escuela Roberto Romero (Ca2) de Salta, manifiesta que desarrollan acciones puntuales centradas en rituales culturales como la Pachamama y en la sexualidad, dado el aumento de embarazos adolescentes. Además, se declara que a todas las acciones que se organiza está invitada la comunidad educativa.

En Sevilla, la comunidad educativa del CEIP San José Obrero (Ca3) de Sevilla, declara que se desarrollan muchas acciones a lo largo del curso, tanto propias del centro escolar como externas de las diversas asociaciones que colaboran y participan con actividades y proyectos específicos sobre las temáticas planteadas, entre las que destaca: la escuela como un espacio abierto para la comunidad, los proyectos específicos de convivencia para toda la comunidad educativa, la visibilización de trabajos referentes a otras culturas expuestos por el centro que enriquece la identidad, etc. En la comunidad educativa del CEIP Andalucía (Ca4) no se reconoce la interculturalidad ni la perspectiva de género, sin embargo, valoran el trabajo que se realiza con las familias desde el centro escolar.

Desde la comunidad educativa de la Escuela Indalecio Gómez (Ca1) de Salta y desde la comunidad educativa de la Escuela Roberto Romero (Ca2) de Salta, se considera positivo y necesario trabajar con materiales didácticos sobre la interculturalidad con perspectiva de género $\mathrm{y}$, asimismo que tenga acceso toda la comunidad educativa, sobre todo, en nivel de enseñanza de infantil y primaria.

Por su parte, la comunidad educativa del CEIP San José Obrero (Ca3) de Sevilla, considera necesario e importante la incorporación de materiales didácticos para trabajar la interculturalidad y la perspectiva de género. Además, destaca que el profesorado hace una gran labor en sus materiales didácticos para que se visibilice la realidad del centro escolar. También están de acuerdo, al igual que en las escuelas salteñas, de trabajar la interculturalidad y perspectiva de género desde etapas de infantil y primaria. La comunidad educativa del CEIP Andalucía (Ca4) de Sevilla, aunque manifiesta que no entiende qué es la interculturalidad y la perspectiva de género, declara que son las acciones con las familias las más importantes que se realizan en el centro escolar, como por ejemplo los talleres, las tertulias literarias, el trabajo de las familias dentro de las clases y las asambleas en las que se tratan temas interesantes donde se intercambian opiniones.

Para una mejora comprensión de los datos obtenidos desde la perspectiva de las comunidades educativas, a continuación, presentamos una tabla explicativa con las principales ideas significativas recopiladas por contexto de estudio.

Tabla 1

Comparativa según las comunidades educativas de los centros escolares por contexto de estudio

\begin{tabular}{|c|c|c|c|c|}
\hline & \multicolumn{4}{|c|}{$\begin{array}{l}\text { PERSPECTIVA DE LA COMUNIDAD EDUCATIVA SOBRE ACCIONES } \\
\text { INTERCULTURALES CON PERSPECTIVA DE GÉNERO }\end{array}$} \\
\hline & \multicolumn{2}{|c|}{ ESCUELAS DE SALTA (Argentina) } & \multicolumn{2}{|c|}{ ESCUELAS DE SEVILLA (España) } \\
\hline & $\begin{array}{c}\text { Caso } 1 \\
\text { Escuela Indalecio } \\
\text { Gómez }\end{array}$ & $\begin{array}{c}\text { Caso } 2 \\
\text { Escuela Roberto } \\
\text { Romero }\end{array}$ & $\begin{array}{c}\text { Caso } 3 \\
\text { CEIP San José } \\
\text { Obrero }\end{array}$ & $\begin{array}{l}\text { Caso } 4 \\
\text { CEIP Andalucía }\end{array}$ \\
\hline $\begin{array}{l}\text { Concepción sobre } \\
\text { interculturalidad } \\
\text { y perspectiva de } \\
\text { género }\end{array}$ & $\begin{array}{l}\text { Se entiende desde la } \\
\text { igualdad de todo el } \\
\text { alumnado, desde la de- } \\
\text { construcción de estereo- } \\
\text { tipos y roles determina- } \\
\text { dos por la cultura que } \\
\text { mantienen las desigual- } \\
\text { dades. }\end{array}$ & $\begin{array}{l}\text { Herramienta didáctica } \\
\text { para adquirir conocimien- } \\
\text { to y crear un mayor vín- } \\
\text { culo con el alumnado que } \\
\text { se encuentra en situación } \\
\text { de desventaja o discrimi- } \\
\text { nación. }\end{array}$ & $\begin{array}{l}\text { Se entiende desde la } \\
\text { igualdad, la inclusión, } \\
\text { la equidad, la convi- } \\
\text { vencia, el respeto de } \\
\text { todo el alumnado, pro- } \\
\text { fesorado y familias. }\end{array}$ & No se especifica. \\
\hline $\begin{array}{l}\text { Acciones inter- } \\
\text { culturales y con } \\
\text { perspectiva de } \\
\text { género }\end{array}$ & $\begin{array}{l}\text { Acciones puntuales rela- } \\
\text { cionadas con la ESI. }\end{array}$ & $\begin{array}{l}\text { Acciones puntuales relacio- } \\
\text { nadas con rituales cultura- } \\
\text { les y con la ESI. }\end{array}$ & $\begin{array}{l}\text { Multitud de acciones } \\
\text { durante el curso, tan- } \\
\text { to internas como ex- } \\
\text { ternas con la colabo- } \\
\text { ración de asociaciones } \\
\text { que trabajan en ambas } \\
\text { materias. }\end{array}$ & $\begin{array}{l}\text { No se reconoce } \\
\text { acciones intercultura- } \\
\text { les y con perspectiva } \\
\text { de género. }\end{array}$ \\
\hline
\end{tabular}




\begin{tabular}{|c|c|c|c|c|}
\hline & \multicolumn{4}{|c|}{$\begin{array}{l}\text { PERSPECTIVA DE LA COMUNIDAD EDUCATIVA SOBRE ACCIONES } \\
\text { INTERCULTURALES CON PERSPECTIVA DE GÉNERO }\end{array}$} \\
\hline & \multicolumn{2}{|c|}{ ESCUELAS DE SALTA (Argentina) } & \multicolumn{2}{|c|}{ ESCUELAS DE SEVILLA (España) } \\
\hline & $\begin{array}{c}\text { Caso } 1 \\
\text { Escuela Indalecio } \\
\text { Gómez }\end{array}$ & $\begin{array}{c}\text { Caso } 2 \\
\text { Escuela Roberto } \\
\text { Romero }\end{array}$ & $\begin{array}{c}\text { Caso } 3 \\
\text { CEIP San José } \\
\text { Obrero }\end{array}$ & $\begin{array}{l}\text { Caso } 4 \\
\text { CEIP Andalucía }\end{array}$ \\
\hline $\begin{array}{l}\text { Materiales didácti- } \\
\text { cos sobre inter- } \\
\text { culturalidad y } \\
\text { perspectiva de } \\
\text { género }\end{array}$ & $\begin{array}{l}\text { No se trabaja con ma- } \\
\text { teriales interculturales } \\
\text { y con perspectiva de } \\
\text { género, pero se conside- } \\
\text { ra necesario y pertinente } \\
\text { para toda la comunidad } \\
\text { educativa. }\end{array}$ & $\begin{array}{l}\text { No se trabaja con ma- } \\
\text { teriales interculturales y } \\
\text { con perspectiva de género, } \\
\text { pero se considera necesa- } \\
\text { rio y pertinente para toda la } \\
\text { comunidad educativa. }\end{array}$ & $\begin{array}{l}\text { Aunque el profesora- } \\
\text { do realiza una gran } \\
\text { labor en el diseño de } \\
\text { sus propios materiales } \\
\text { didácticos ajustados a } \\
\text { la realidad del centro } \\
\text { escolar, se considera } \\
\text { necesarios y pertinen- } \\
\text { tes. }\end{array}$ & $\begin{array}{l}\text { Aunque no se en- } \\
\text { tiende qué es la in- } \\
\text { terculturalidad y la } \\
\text { perspectiva de géne- } \\
\text { ro, se considera nece- } \\
\text { sario y pertinente tra- } \\
\text { bajar con las familias. }\end{array}$ \\
\hline
\end{tabular}

Fuente. Elaboración propia a partir de la información obtenida de los grupos de discusión a las comunidades educativas de cada centro educativo.

\section{Comparación de la Visión de las Comunidades Educativas ante las Acciones Interculturales con Pers- pectiva de Género}

Entre los resultados más significativos de la comparativa sobre la percepción del profesorado sobre las acciones interculturales con perspectiva de género de los centros escolares de Salta y Sevilla encontramos diferencias significativas en dos de sus ítems, como se puede observar en la siguiente tabla $\mathrm{n}^{\circ} 2$.

\section{Tabla 2}

Acciones interculturales con perspectiva de género en el centro escolar

\begin{tabular}{|c|c|c|c|c|c|c|c|}
\hline & \multicolumn{7}{|c|}{ Grado de acuerdo } \\
\hline \multirow{5}{*}{$\begin{array}{c}\text { Ítems } 93 \text { y } 94 \\
\text { El profesorado centra su } \\
\text { actuación desde las car- } \\
\text { acterísticas propias del } \\
\text { alumnado y su familia }\end{array}$} & & & Nada \% & Poco \% & Algo \% & Bastante \% & Md \\
\hline & \multirow[t]{2}{*}{ Salta } & Ca1 & 14,3 & 14,3 & 42,9 & 28,6 & 3,0 \\
\hline & & $\mathrm{Ca2}$ & - & 60,0 & 40,0 & - & 2,0 \\
\hline & \multirow[t]{2}{*}{ Sevilla } & $\mathrm{Ca3}$ & - & - & 36,4 & 63,6 & 4,0 \\
\hline & & Ca4 & - & - & 11,1 & 88,9 & 4,0 \\
\hline \multirow{4}{*}{$\begin{array}{c}\text { El profesorado centra } \\
\text { su actuación desde las } \\
\text { características propias } \\
\text { del contexto sociocultural } \\
\text { del centro escolar }\end{array}$} & \multirow[t]{2}{*}{ Salta } & Ca1 & - & 14,3 & 14,3 & 71,4 & 4,0 \\
\hline & & $\mathrm{Ca2}$ & 20,0 & 40,0 & 20,0 & 20,0 & 2,0 \\
\hline & \multirow[t]{2}{*}{ Sevilla } & $\mathrm{Ca3}$ & - & - & 27,3 & 72,7 & 4,0 \\
\hline & & Ca4 & - & - & 11,1 & 88,9 & 4,0 \\
\hline
\end{tabular}

Fuente. Elaboración propia a partir de los datos obtenidos de la encuesta al profesorado.

En el primero de ellos, el ítem 93 El profesorado centra su actuación desde las características propias del alumnado y su familia $(x 2=14,716$; g.l. $=3 ; p=0.002)$ la significación la hallamos en la Escuela Roberto Romero (Ca2-60\%) de Salta en la que el profesorado indica no estar de acuerdo con dicha afirmación, mientras que el profesorado tanto de los centros escolares de Sevilla, el CEIP San José Obrero (Ca3-100\%) y el CEIP Andalucía (Ca4-100\%), como de la Escuela Indalecio Gómez (Ca1-71,5\%), sí señala que está de acuerdo que su actuación se centra desde las características propias del alumnado y su familia.

En función a este dato reflexionamos, que más de la mitad del profesorado de la Escuela Roberto Romero (Ca2-60\%) haya considerado principal el contenido curricular que los intereses, capacidades, competencias y actitudes del alumnado en su proceso de aprendizaje, circunstancia planteada como dificultad y obstáculo a la hora de trabajar dichas temáticas. Es decir, dentro del equipo docente hay más de la mitad del profesorado que dirige su docencia a que el alumnado adquiera más contenido teórico, vinculado a la tradicional educación academicista, que competencias y capacidades más actitudinales, de reflexión y visión crítica dada a partir de una educación más socioeducativa. 
En segundo lugar, acerca de las acciones interculturales con perspectiva de género en el centro escolar, hallamos diferencia significa en el ítem 94 El profesorado centra su actuación desde las características propias del contexto sociocultural del centro escolar ( $x 2=9,446 ; \mathrm{g} . \mathrm{l} .=3 ; p=0.024)$, al igual que en el ítem anterior, como se puede observar en la tabla $n^{\circ} 2$, la significación es la misma, siendo el profesorado de la Escuela Roberto Romero (Ca2-60\%) de Salta, el que indica no estar de acuerdo con la afirmación, frente a la otra escuela salteña, Escuela Indalecio Gómez (Ca1-85,7\%), más los dos centros escolares de Sevilla, el CEIP San José Obrero (Ca3-100\%) y el CEIP Andalucía (Ca4-100\%) que sí indica el profesorado de cada institución educativa estar de acuerdo que centra su actuación desde las características propias del contexto sociocultural en el que se encuentra el centro escolar.

Como se ha mencionado, esta significación quizás sea debida a las tensiones surgidas en función al tipo de docencia que el profesorado quiere impartir, considerando primordial más la adquisición de conocimiento teórico, que prácticos y actitudinales en el proceso de enseñanza-aprendizaje del alumnado. Cabe destacar que, en esta escuela salteña (Ca2-Escuela Roberto Romero), concretamente, no se trabaje la diversidad cultural ni la perspectiva de género porque cierto profesorado no las considere importantes para su docencia.

En relación con la formación y la práctica del profesorado (véase tabla nº 3), así como con las acciones interculturales con perspectiva de género en los centros escolares de Sevilla y Salta, hacemos hincapié en dos de sus ítems en los que hallamos significación.

En el ítem 103 El profesorado ajusta su programación anual según los diferentes ritmos de aprendizajes, capacidades, necesidades e intereses del alumnado $(x 2=9,422 ; g . l .=3 ; p=0.024)$ encontramos diferencia significativa en el Caso 2 - Escuela Roberto Romero de Salta, donde un 50\% del profesorado india que está poco de acuerdo en dicha afirmación. Este ítem se relaciona directamente con los ítems 93 y 94, anteriormente mencionados en la tabla $n^{\circ} 2$. Es decir, la mitad del profesorado de la Escuela Roberto Romero (Ca250\%) señala que no está de acuerdo en que se ajuste su programación anual según los diferentes ritmos de aprendizajes, capacidades, necesidades e intereses del alumnado, ni tampoco que centre su actuación en las características propias del alumnado, las familias y el contexto sociocultural de la institución educativa. En este mismo sentido, la mitad del profesorado encuestado de la Escuela Roberto Romero (Ca2) no considera necesario hacer ajustes ni en su programación ni en su actuación docente debido quizás a una forma de trabajar más rígida en contenido académico, que en la adquisición de competencias y capacidades socioeducativas del alumnado.

\section{Tabla 3}

Profesorado, formación y acciones interculturales con perspectiva de género

\begin{tabular}{|c|c|c|c|c|c|c|c|}
\hline \multirow[b]{2}{*}{ Ítems 103 y 104} & \multicolumn{7}{|c|}{ Grado de acuerdo } \\
\hline & & & Nada \% & Poco \% & Algo \% & Bastante \% & Md \\
\hline \multirow{4}{*}{$\begin{array}{l}\text { El profesorado ajus- } \\
\text { ta su programación } \\
\text { anual según los } \\
\text { diferentes ritmos } \\
\text { de aprendizajes, ca- } \\
\text { pacidades, necesi- } \\
\text { dades e intereses } \\
\text { del alumnado }\end{array}$} & \multirow[t]{2}{*}{ Salta } & Ca1 & - & 28,6 & 28,6 & 42,9 & 3,00 \\
\hline & & Ca2 & - & 50,0 & 16,7 & 33,3 & 2,50 \\
\hline & \multirow[t]{2}{*}{ Sevilla } & $\mathrm{Ca3}$ & - & - & 18,2 & 81,8 & 4,00 \\
\hline & & Ca4 & - & - & 11,1 & 88,9 & 4,00 \\
\hline \multirow{4}{*}{$\begin{array}{c}\text { Sobre las medi- } \\
\text { das de educación } \\
\text { intercultural con } \\
\text { perspectiva de } \\
\text { género, se conside- } \\
\text { ra mejor trabajarlas } \\
\text { a partir de los ciclos } \\
\text { de secundaria }\end{array}$} & \multirow[t]{2}{*}{ Salta } & Ca1 & 14,3 & 71,4 & 14,3 & - & 2,00 \\
\hline & & Ca2 & 40,0 & 60,0 & - & - & 2,00 \\
\hline & \multirow[t]{2}{*}{ Sevilla } & Ca3 & 72,7 & 27,3 & - & - & 1,00 \\
\hline & & Ca4 & 88,9 & - & - & 11,1 & 1,00 \\
\hline
\end{tabular}

Fuente. Elaboración propia a partir de los datos obtenidos de la encuesta al profesorado. 
En segundo lugar, con respecto al ítem 104 sobre las medidas de educación intercultural con perspectiva de género, se considera mejora trabajarlas a partir de los ciclos de secundaria $(x 2=9,195 ;$ g.l. $=3$; $p=0.027$ ) encontramos diferencia significa en dos de los casos de estudios. Exponemos, por una parte, que el profesorado de todos los centros escolares de Sevilla y Salta no está de acuerdo en señalar que las medidas de educación intercultural con perspectiva de género sea mejor considerarlas a partir de los ciclos de secundaría, tal y como también se expone en las diferentes comunidades educativas. Es decir, en todos los centros escolares, el profesorado está de acuerdo en que las acciones interculturales con perspectiva de género se consideran trabajarlas desde niveles de enseñanzas de infantil y primaria. Esto nos lleva a reflexionar que la interculturalidad con perspectiva de género sea un enfoque importante para trabajar desde edades tempranas para luchar contra situaciones de desigualdad, discriminación y/o violencia por cuestiones culturales y de género entre el alumnado, posibilitando una formación inclusiva hacia valores positivos de la diferencia y valores democráticos y cívicos, donde el alumnado tenga capacidad de pensamiento crítico y mirada positiva ante dichas situaciones injustas.

Sin embargo, por otra parte, el profesorado tanto en la Escuela Indalecio Gómez (Ca1-14,3\%) de Salta y el CEIP Andalucía (Ca4-11,1\%) indica con porcentaje bajos, en cada caso, que sí está de acuerdo que las medidas interculturales con perspectiva de género deban considerarse a partir de los niveles de secundaria. Este dato significante, aunque con bajos porcentajes, puede ser debido a que cierto profesorado considera no relevante trabajar este enfoque de gestión de la diversidad cultural y la perspectiva de género en escuelas de infantil y primaria, dejando la reflexión de dichas temáticas para alumnado con mayor capacidad de reflexión y compresión, como es el alumnado de secundaria.

Ante esta posibilidad, no se está de acuerdo, ya que se considera que es en el proceso de socialización de los/as niños, niñas y adolescentes donde se debe hacer hincapié en el trabajo de dichas temáticas para eliminar posteriores pensamientos y actitudes pesimistas, racistas y sexistas por cuestiones diferenciales en función a la cultura y/o al género.

\section{Aporte conclusivo}

Tras los datos obtenidos, debemos reflexionar qué estrategias socioeducativas pueden caracterizar a las escuelas para denominarlas centros interculturales con perspectiva de género.

Ante lo expuesto, podemos avanzar a partir del conocimiento, entendimiento y valoración que se tiene por parte de toda la comunidad educativa escolar sobre las temáticas planteadas, tal y como se recoge con esta investigación. Asimismo, fomentar en las instituciones educativas la importancia de trabajar desde un enfoque intercultural con perspectiva de género tanto dentro como fuera del marco institucional, en la que se cuente con todos y todas los/as agentes socializadores.

Como resultados significativos obtenemos que solo en uno de los centros escolares analizados, el CEIP San José Obrero de Sevilla (Ca3), se trabaja de forma continuada la interculturalidad con perspectiva de género, siendo ésta abordada, en su mayoría, de forma general y puntual, en su mayoría. Asimismo, resaltamos el perfil institucional del CEIP San José Obrero (Ca3) como ese centro escolar que más se acerca a este enfoque socioeducativo a nivel teórico-práctico con carácter pedagógico.

Sin embargo, la interculturalidad con perspectiva de género se recoge más bien como un planteamiento teórico, donde la práctica real, efectiva y continuada resulta difícil de ver en las instituciones educativas analizadas, especialmente en las escuelas salteñas y el CEIP Andalucía de Sevilla (Ca4), que entendemos que sea por la idiosincrasia propia del centro. Por ello, como profesionales de la educación debemos promover esta idea teórica-práctica, así como fomentarla como filosofía escolar y como modelo socioeducativo a implementar (Rodriguez, 2018). En cambio, esto solo es posible con concienciación sobre las temáticas, formación para toda la comunidad educativa (Carrasco, 2015) y participación de agentes socioeducativos, como los/as educadores/as sociales, en todos los niveles educativos y de las administraciones, tanto públicas como privadas, que en definitiva son las que crean y diseñan, a nivel políticas, los programas que hoy en día se denominan "interculturales y con perspectiva de género". 
Con ello, con implicación, coordinación y profesionalidad interdisciplinar podremos construir y transformar las escuelas hacia la interculturalidad con perspectiva de género desde una perspectiva socioeducativa, ya que, como principios básicos en toda escuela con diversidad, es imprescindible tener en cuenta el carácter social, así como conocer y respetar a cada alumnado como único (Ballesteros et al. 2014). Es aquí donde profundizamos en la extensión social del proceso educativo, puesto que la educación se consolidad en sociedad y reflexionando que todo sistema educativo está influenciado por factores políticos y económicos, herencia ideológica histórica, paradigma pedagógico, etc. En fin, particularidades propias y condicionantes de cada institución educativa (Egido, 2011).

En cambio, nos seguimos preguntando quién es el o la responsable de una educación intercultural con perspectiva de género capaz de abordar a toda la comunidad educativa en las mismas condiciones de derechos, deberes y obligaciones, ante las problemáticas antirracistas, sexistas, insolidarias, injustas... que mantiene nuestra sociedad y que se refleja en los centros escolares. Pues, de acuerdo con Caparrós (2012) la educación debe ayudar a sensibilizar y a crecer con una moral y ética hacia los derechos humanos, alcanzando que toda la comunidad educativa se parte de su transformación en actitudes, pensamientos y conocimiento para el respeto del bienestar común.

Como derecho de y para la ciudadanía, la educación debe presidir el principio de igualdad, independientemente de cuestiones culturales y de género. Por ello, es necesario renovar las actuales metodologías docentes, así como innovar en estrategias interculturales con perspectiva de género para conseguir la plenitud educativa para todos y todas sus protagonistas. Así, todo sistema educativo debe garantizar una escuela inclusiva que cuente con todos los recursos posibles para hacer efectiva la finalidad de la educación intercultural con perspectiva de género.

Apostamos por una educación inclusiva desde la participación y en una educación para la igualdad, pues de acuerdo con Calvo (2009, p. 49) esta modalidad educativa "implica cambiar la estructura, funcionamiento y propuesta pedagógica de los centros para dar respuesta a las necesidades educativas de todos de forma que todos tengan éxito en su aprendizaje y participen en igualdad de condiciones". En la misma línea, la escuela debe ser ese espacio individual y colectivo en el que poder soñar y construir la necesaria cohesión social que guie el trabajo desde las diferentes instituciones educativas y sociales (Lorenzo, 2012). En este sentido, la escuela necesita un impulso en los niveles de participación y compromiso por parte de todos/as sus protagonistas para esa construcción crítica, interpretativa, actuante, transformadora y de responsabilidad compartida con la finalidad de diseñar o renovar estrategias socioeducativas para la interculturalidad con perspectiva de género, como puede verse en la siguiente figura.

\section{Figura 2}

\section{Estrategias socioeducativas para la interculturalidad con perspectiva de género}

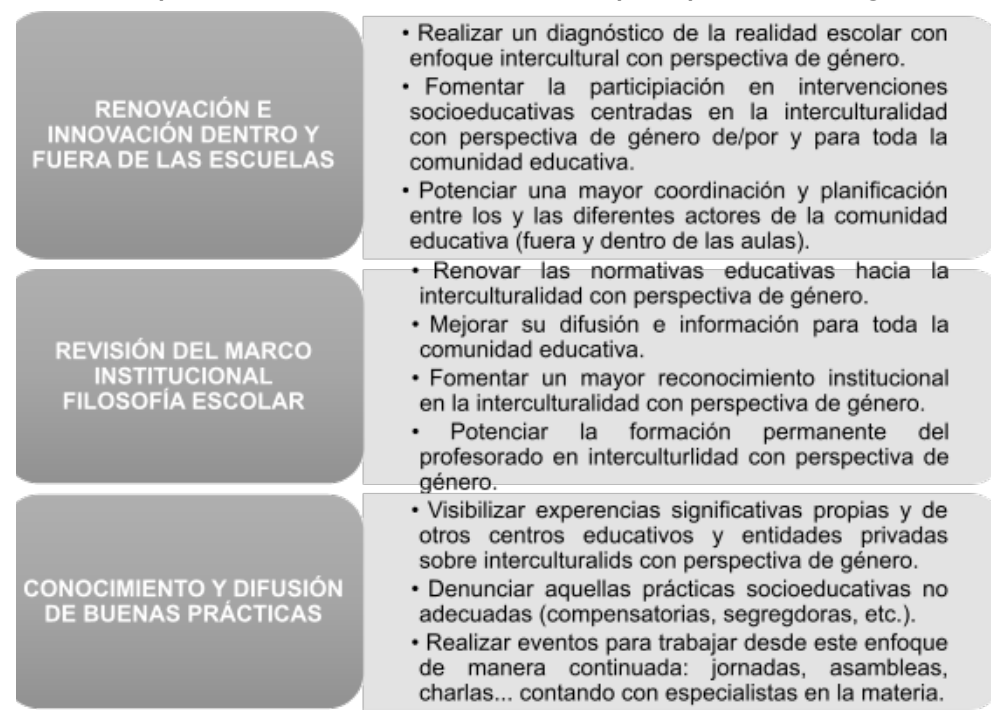

Fuente. Elaboración propia. 
En definitiva, a partir de un exhaustivo análisis de la realidad interdisciplinar y desde la interculturalidad con perspectiva de género podemos reforzar una conciencia y sensibilidad mayor para atender y gestionar la diversidad cultural y de género en las escuelas con el fin de visibilizar las desigualdades y discriminaciones, que sufren no solo el alumnado, sino toda la comunidad educativa.

\section{Referencias}

Aguado, T. (2004). Investigación en educación intercultural. Revista Educatio Siglo XXI, 22, 39-57.

Álvarez, J. L. (2008). La investigación en educación intercultural. En A. Pantoja, T.J. Campoy, A. Jiménez y C. Villanueva (Coord.) El carácter universal de la educación intercultural. Actas de las I Jornadas Internacionales y VI sobre Diagnóstico y Orientación. Jaén: Servicio de Publicaciones de la Universidad.

Ariño, A. (2009). Estilos de aculturación y encrucijadas de la diversidad cultural. Papers, 94, 115-137.

Ayuste, A. (2011). La educación transformadora en la pedagogía contemporánea. Revista Crítica, 972, 16-20.

Ballesteros, B., Aguado, T. y Malik, B. (2014). Escuelas para todos: diversidad y educación obligatoria. Revista Electrónica Interuniversitaria de Formación del Profesorado, 17 (2), 93-107.

Barbieri, C. y Ferede, M.K. (29 de junio de 2020) Un futuro con el que todos podamos vivir: cómo la educación puede abordar y erradicar el racismo. LABO de Ideas de Los Futuros de la Educación de la UNESCO.

Besalú, X. (2002). Diversidad cultural y educación. Síntesis.

Borrero, R. (2012). Educación para todos y todas. Análisis del modelo educativo social para una Educación Intercultural. Revista Educatio siglo XXI, 30 (2), 333-364.

Calvo, M.I. (2009). Participación en la Comunidad. En Sarto, M.P. y Venegas, M.E. (Coord.), Aspectos clave de la Educación Inclusiva (pp. 41-58). Salamanca: INICO.

Caparrós, F. J. (2012). Apostillas al capítulo III del Código Deontológico del Educador Social. RES. Revista de Educación Social, 15, 1-5.

Carrasco, C. (2015). Discurso de futuros docentes acerca de la diversidad intercultural. Papers, 100 (2), 155-172.

Corbetta, P. (2007). Metodología y técnicas de investigación social. McGraw-Hill.

Del Canto, E. y Silva, A. (2013). Metodología cuantitativa: abordaje desde la complementariedad en Ciencias Sociales, Revista Ciencias Sociales, 3 (141), 25-34.

Egido, I. (2011). Escuelas y educación para la transformación social. Revista Crítica, 972, 16-20.

Lorenzo, Ma A. (2012). Del inconformismo a la innovación pasando por una mejora en educación. En D. Cobos Sanchíz, E. López Meneses, A. Jaén Martínez, A.H. Martín Padilla y L. Molina García (Dirs), Actas del I Congreso Virtual Internacional sobre Innovación Pedagógica y Práxis Educativa, pp. 382-392. España: AFOE.

Medina, E. (2019) Ecofeminismo e interculturalidad. En Feminismo/s, 34, pp. 199-214. Dosier monográfico: Estado actual de la investigación en Literatura francesa y Género: balance y nuevas perspectivas / État présent de la recherche en Littérature française et Genre: bilan et nouvelles perspectives, coord. Ángeles Sirvent Ramos, DOI: 10.14198/fem.2019.34.09 
Osuna, C. (2012). En torno a la educación intercultural. Una revisión crítica. Revista de Educación, 358, 38-58

Rodríguez, H., Gallego, B., Sansó, C., Navarro, J. L., Velicias y M., Lago, M. (2011). La educación intercultural en los centros escolares españoles. Revista Electrónica Interuniversitaria de Formación del Profesorado-REIFOP, 14 (1), 101-112.

Rodríguez, R. (2017). Diversidad cultural y perspectiva de género en centros educativos de infantil. [Tesis Doctoral. Universidad Pablo de Olavide. España].

Rodríguez, R. (2018) Aportes para el desarrollo de prácticas interculturales con perspectiva coeducativa en centros escolares. La educación social como herramienta de cambio en el ámbito reglado. En clave Pedagógica. No 14, p. 51-59.

Ruiz, C. (2017). Estrategias para educar en y para la igualdad: coeducar en los centros. ATLÁNTICAS-Revista Internacional de Estudios Feministas, 2 (1), 166-191.

Subirats, M. (2016). De los dispositivos selectivos en la educación: el caso del sexismo. Revista de Sociología de la Educación RASE, vol. 9, n¹, pp. 22-36.

Tomé, A. (2011). Los valores que sustentan la coeducación. El valor de la autonomía en niñas y niños de 6 años. EMAKUNDE - Instituto Vasco de la Mujer. Programa coeducativo para la igualdad, el respeto y la no-violencia. Seminario de Formación con el profesorado del Proyecto NAHIKO! 1-2. Vitoria-Gasteiz, pp. 1-11.

Valles, M. S. (1999). Técnicas cualitativas de investigación social. Reflexión metodológica y práctica profesional. Síntesis. 